e-migrinter

e-Migrinter

12 | 2014

Les chiffres de l'immigration : un regard critique sur les statistiques

\title{
Victor Piché, Les théories de la migration
}

\section{Celio Sierra-Paycha}

\section{OpenEdition}

Journals

Édition électronique

URL : https://journals.openedition.org/e-migrinter/348

DOI : 10.4000/e-migrinter.348

ISSN : 1961-9685

Éditeur

UMR 7301 - Migrinter

Édition imprimée

Date de publication : 15 octobre 2014

Pagination : 108-111

ISSN : 1961-9685

\section{Référence électronique}

Celio Sierra-Paycha, « Victor Piché, Les théories de la migration », e-Migrinter [En ligne], 12 | 2014, mis en ligne le , consulté le 20 mai 2021. URL : http://journals.openedition.org/e-migrinter/348 ; DOI : https:// doi.org/10.4000/e-migrinter.348 


\section{Piché, Victor (éd.) (2013) Les théories de la migration, Paris, INED, 536 p. (Les Manuels, série Les Textes fondamentaux)}

\section{Celio Sierra-Paycha}

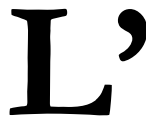

objectif premier de la collection Textes fondamentaux est de rassembler certains des textes fondateurs de la démographie ayant un fort contenu théorique, afin de pallier le manque de travaux théoriques qui, selon ses détracteurs, caractériserait la discipline. Le deuxième objectif est de les rendre accessible à un public francophone de "chercheurs et d'étudiants en démographie» (p. 9), en traduisant ces textes fondateurs. Cet opus consacré aux théories de la migration répond à ces deux objectifs. À partir du recensement de publications fondatrices datant de la période de l'après-guerre jusqu'au début des années 2000, Victor Piché rassemble vingt et un textes organisés en trois parties: les origines et les causes des migrations, les effets de la migration et les politiques migratoires.

Les deux premiers chapitres offrent des clés de lecture afin d'articuler ces différentes théories. Le chapitre introductif écrit par V. Piché situe les différents éléments de ce corpus dans l'histoire plus générale des idées en sciences économiques et sociales (approches néoclassique, fonctionnaliste, marxiste, théories du genre, nouvelle économie). Alan Simmons (chapitre 2) constate que depuis Ravenstein au XIX $^{\text {ème }}$ siècle, les théories des migrations constituent un corpus théorique hétérogène. Cette hétérogénéité s'explique par le contexte historique d'apparition et de production de ces théories, ainsi que par la divergence des phénomènes observés. Les théories fonctionnalistes sont héritières d'une vision dix-neuvièmiste des migrations. Celles-ci empruntent au monde industriel les métaphores de la mécanique et du «flux ». Les flux sont actionnés par des facteurs d'attraction et de répulsion. Les théories interactionnistes les plus récentes quant à elles, empruntent à la cybernétique ses termes de «circuits", de « réseaux » et de «voies de communication». Ces théories sont opérantes pour l'étude des mouvements propres aux sociétés post-industrielles.

La succession des chapitres suit de manière générale un ordre chronologique. Une lecture linéaire permet de dérouler l'histoire des théories de la migration à travers ces différents champs théoriques mais aussi disciplinaires (Géographie, Démographie, Économie, Sociologie, Sciences Politiques).

Les chapitres 3, 4 et 5 proposent des approches micro-individuelles de la migration ayant vocation à établir les lois de la migration. L'économiste Larry Sjaastad (chapitre 3) de l'École de Chicago propose de lire les phénomènes migratoires à la lumière de "taux nets" prenant en compte les départs et les arrivées, contrairement aux «taux bruts». Ces taux de migration sont fonctions de coûts, de bénéfices monétaires (augmentation des dépenses quotidiennes, coûts de transports) et non-monétaires (coûts d'opportunité liés à la perte d'emploi, coût psychique du déracinement). Everett Lee (chapitre 4) entend expliquer les migrations par une série de facteurs, facteurs liés aux lieux de départ et d'installation, facteurs individuels, obstacles intermédiaires. Le géographe américain Wilbur Zelinsky (chapitre 5) pose les jalons d'une « théorie de la transition de la mobilité » qui articulerait l'échelle temporelle et l'échelle spatiale. Ce dernier décompose la transition de la mobilité en cinq phases. Après une première phase où le niveau de mobilité est faible et 
les niveaux de mortalité et de fécondité sont élevés, les mobilités rurale-rurale, mais aussi, rurale-urbaine et internationales augmentent dans une seconde phase, tandis que la population croît grâce à des taux de fécondité élevés, et des taux de mortalité désormais faibles. Lors de la troisième phase, le taux de fécondité rejoint celui de la mortalité et la migration résidentielle diminue au profit d'une augmentation de la circulation. Ce phénomène se poursuit dans la quatrième phase, où les niveaux de mortalité, de fécondité et de migration résidentielle stagnent tandis que la circulation augmente. Une dernière phase anticipatrice correspondrait à une réduction de la mobilité, et plus particulièrement à une réduction de la circulation.

Alors que ces premiers textes contiennent des approches micro, une autre série de chapitres $(6,7,8,13$, et 15$)$ propose une lecture plus systémique des migrations. Akin Mabogunje (chapitre 6) étudie la migration rurale-urbaine africaine non comme une simple mécanique d'attraction et de répulsion, mais comme un "système ", « un ensemble complexe composé d'éléments en interaction» (p. 153). Ce système comprend un environnement social, économique, technologique rapprochant socialement, symboliquement et physiquement les campagnes des villes, environnement, qui agit sur les potentiels migrants, avec des stimuli. Ce système global est régi par des sous-systèmes ruraux, tels que la famille (départ des cadets plutôt que des aînés) la communauté villageoise, mais aussi des sous-systèmes urbains (législations et opportunités d'emploi) régulant les départs et les arrivées de migrants.

Le sociologue du travail Michael Burawoy (chapitre 7), marxiste et membre de l'École de Chicago, dépasse l'analyse classique des migrations en termes de causes et de conséquences, pour une analyse critique des institutions régulant les flux de main d'œuvre. Les fonctions d'entretien et de reproduction de la main d'œuvre sont habituellement remplies par les mêmes institutions (famille, État, travail domestique); dans le système de la main d'œuvre migrante, ces fonctions sont dissociées (la main d'œuvre née au Mexique travaille aux États-Unis), tout en étant interdépendantes (mouvements entre Mexique et États-Unis). Le travailleur reproductif est ainsi rendu dépendant du revenu du travailleur productif, tandis que l'État, privant le travailleur productif de statut juridique et politique durable sur son lieu de travail (avec les «barrières de couleur» en Afrique du Sud par exemple) le rend dépendant du travailleur reproductif (sa famille). L'article de Stephen Castles et de Godula Kosack (chapitre 13) privilégie également une approche marxiste de la migration des travailleurs, autour de deux concepts centraux, permettant de comprendre la domination des masses laborieuses, à savoir, l'armée industrielle et l'aristocratie ouvrière. Dans le contexte du «capitalisme de monopole organisé » et celui de plein emploi des Trente glorieuses, les migrants du «tiers monde » apparaissent comme la nouvelle armée de réserve «latente» et les ouvriers nationaux remplissent la fonction «d'aristocratie ouvrière ». La séparation objective entre ouvriers natifs et ouvriers immigrés dans le processus économique de production est renforcée par une division sociale et symbolique, par la langue, la ségrégation résidentielle et le statut. La sociologue Saskia Sassen (chapitre 8) s'interroge sur les conditions d'apparition d'une demande de main d'œuvre dans les grands centres urbains des pays très industrialisés où coexistent une demande de travail hautement qualifié (dirigeants de multinationales) et une demande de travail peu qualifié (femmes de ménage) au sein de la filière de production de "services très spécialisés ». L'économiste George Borjas (chapitre 15) réfute l'idée reçue d'un impact négatif de la migration sur les natifs, en affirmant que s'il y a bien un impact des nouveaux migrants sur le revenu des migrants déjà installés, les effets de 
l'immigration en général - et des immigrés clandestins en particulier - sur les revenus des natifs, sont négligeables.

Le recueil de V. Piché fait état d'un tournant épistémologique dans les années 1980 où apparaissent les niveaux méso d'observation et d'analyse des migrations. Les économistes Oded Stark et David Bloom (chapitre 10) affirment que la décision de migrer n'est pas individuelle. Il s'agit d'un contrat implicite entre les migrants et les non-migrants. L'interdépendance mutuelle entre ces groupes intervient dans le calcul de la stratégie des migrants. Une fois sur le marché du travail, la force du travailleur migrant est son capital de réseau et de parenté ; dans les lieux d'origine, la diffusion de l'information sur les bénéfices de la migration a un impact sur la taille des flux de départs. Monica Boyd (chapitre 11) tente de conceptualiser le rôle central des réseaux et de la famille dans les migrations : le réseau social est un concept qui permet d'articuler le niveau micro, sous-socialisé, et le niveau macro, sur-socialisé, car les réseaux transmettent et façonnent l'influence des structures sur les individus, les familles et les ménages. Les liens sociaux transmettent des informations relatives aux lieux d'arrivée et offrent des ressources en matière d'installation. Les migrations sont donc ici vues comme un produit social, non comme le résultat exclusif de décisions individuelles prises par des acteurs individuels, ni comme celui des paramètres économiques et politiques, mais plutôt comme le produit de l'interaction de tous ces facteurs.

Les conclusions du sociologue Alejandro Portes et de Kenneth Wilson (chapitre 16) à partir de l'enquête longitudinale menée sur 590 Cubains primoarrivants à Miami de 1973 à 1977, montrent que les migrants peuvent s'insérer dans des enclaves migrantes, c'est-à-dire des concentrations d'entreprises détenues par des migrants ayant accumulé du capital avant et pendant la migration et qui embauchent des migrants compatriotes en valorisant leur capital humain, en leur offrant des perspectives de mobilité sociale. L'intégration de ces approches méso d'observation renouvelle les perspectives de recherche sur les migrations internationales : Mirjana Morokvasic (chapitre 9) fait un bilan des études de genre portant dans ce champ de recherche en 1984, les économistes indiens Oberaï et Manmohan Singh (chapitre 14) s'intéressent aux effets macroéconomiques des transferts monétaires familiaux des migrants à partir d'une enquête du Bureau International du Travail dans le Pendjab indien; Douglas Massey (chapitre 12) plaide pour une approche multiniveaux (individu, ménage et communauté) des migrations.

Une dernière série de chapitres est consacrée aux politiques migratoires. Le politiste canadien Joseph Carens (chapitre 19) teste les fondements théoriques et moraux de la fermeture des frontières à partir de trois cadres théoriques et idéologiques (étatiste, rawlsien et utilitariste). Ce dernier constate l'aporie théorique que constitue la fermeture des frontières. Pourtant, comme le montre Bimal Ghosh (chapitre 20), les États persistent à mettre en place des politiques migratoires restrictives, alors que les pays européens ont retiré d'immenses avantages des flux d'émigration et d'immigration non restreints. Le démographe français Georges Tapinos (chapitre 21) observe que l'impact économique de la migration clandestine généré par ces politiques restrictives est surtout positif pour les employeurs. Les migrants clandestins s'implantent dans des économies souterraines marquées par la désalarisation, la sous-traitance et la flexibilité du travail ; l'impact sur l'emploi et sur le travail des natifs est donc limité et le bilan social des migrations clandestines est plutôt positif pour les nationaux. Selon Stephen Castles (chapitre 18), les États qui tendent à devenir multiculturels devraient redéfinir leur politique migratoire en précisant le concept de solidarité 
internationale. Il affirme d'ailleurs que ces migrations ne peuvent se comprendre que dans un cadre global et par-delà les distinctions entre les différents types de migrations qui n'ont plus de sens. Aristide Zolberg, Astri Suhrke et Sergio Aguayo (chapitre 17) envisagent le phénomène des réfugiés dans une perspective transnationale. Bien que le droit international définisse les réfugiés comme des personnes subissant une crainte de persécution fondée dans leur pays d'origine et franchissant une frontière internationale, les auteurs montrent que la dimension internationale réside aussi dans le processus de formation des réfugiés. En effet, une intervention militaire extérieure dans le pays d'origine ou encore une guerre civile sous influence de puissances étrangères peuvent occasionner un flux de réfugiés; de manière indirecte, la politique migratoire des pays d'accueil peut agir comme force d'attraction de ces flux, enfin, une politique d'asile peut être utilisée comme arme diplomatique contre un pays ennemi persécuteur.

On constate un léger déséquilibre disciplinaire dans cet ouvrage: six articles d'économie, six articles de sociologie, quatre articles de sciences politiques, quatre de démographie et deux articles de géographie. Ce déséquilibre tient sans doute au poids réel de certains écrits dans la théorie des migrations, mais peut être aussi interprété comme le produit d'un effet de réseau dans la constitution du corpus, au détriment d'approches spatiales du phénomène migratoire. À titre d'exemple, aucun article n'est issu de la Revue Européenne des Migrations Internationales dont l'apport aux théories migratoires est pourtant mentionné dans une note de bas de page (p. 17).

Cet ouvrage permet d'ébaucher une histoire de la pensée des théories de la migration, tout en rendant accessible à un public francophone des références de première main à travers des auteurs qui apparaissent fréquemment dans les bibliographies. La lecture de ce recueil complète donc celle d'autres ouvrages à vocation similaire comme le Bilan des travaux sur la circulation migratoire (Doraï, Hily et $\mathrm{Ma}$ Mung, 1998) ${ }^{1}$.

Celio Sierra-Paycha Doctorant en géographie Migrinter-Université de Poitiers

INED celio.sierra@gmail.com
${ }^{1}$ Doraï, K. ; Hily, M.A. ; Ma Mung, E. (1998) Bilan des travaux sur la circulation migratoire. Rapport à la Direction de la Population et des Migrations, Ministère de la Solidarité et de l'Emploi, Poitiers, Migrinter, 146 p. 
$2 \quad \mathbf{n}^{\circ} 12 \quad 2014$

(e-migrinter 